\title{
Lithography materials guidelines
}

\author{
Harry J. Levinson \\ $\mathrm{JM}^{3}$ Co-Editor-in-Chief
}

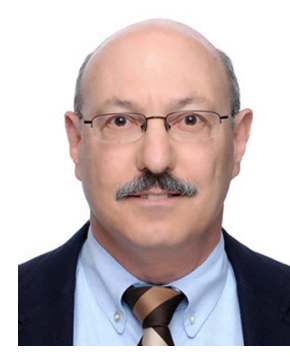

Reproducibility has long been considered a critical element of the scientific method, and the ability to confirm published results depends on sufficient information being disclosed in scientific papers to enable the reproduction of the original results by other researchers. On the other hand, for publications in lithography, it has generally not been expected that suppliers of materials and equipment will disclose proprietary information that provides a competitive advantage. Until this journal, the Journal of Micro/Nanolithography, MEMS and MOEMS $\left(\mathrm{JM}^{3}\right)$, was established, the vast majority of publications in our field consisted of conference proceedings, which are subject to different norms than publications in peer-reviewed journals for all technical fields. It has always been expected that the standards for papers published in $\mathrm{JM}^{3}$ should be stricter than those for conference proceedings papers, but such standards have not yet been articulated, making it difficult for potential authors to know whether their papers will be considered acceptable or not for publication in $\mathrm{JM}^{3}$.

To address this issue, several of the editors of this journal have devised a set of standards for papers concerned with materials for lithography. These guidelines are intended to strike a balance between providing readers of $\mathrm{JM}^{3}$ with information important for the use of these materials and the practical necessity for companies to safeguard their critical proprietary information.

Guidelines for publications in $\mathrm{JM}^{3}$ regarding materials for lithography:

- We encourage full disclosure of formulation and processing information.

- If it's a proprietary formulation, state: proprietary formulation.

- If it's a commercial product: state its name.

- Minimum formulation and process conditions disclosure should include:

○ Resist type: chemically amplified (CAR), metal-containing (MCR), or if neither, an appropriate qualitative composition description

- Resist tonality: positive tone or negative tone

- Developer type: water-based (and normality) or solvent-based

- Resist film thickness

- If MCR, disclose the elemental metal/metals

- If the resist is known to have an unusual optical density, state the OD

- If the study uses several resist variants, state what is varied

- Substrate: spin-on or deposited layer or bare silicon

- If pertinent to the analysis and not disclosed in the manuscript, the $\mathrm{JM}^{3}$ editor may require authors to provide additional generic formulation information. (Examples: PAG, polymer bound PAG, quencher, photodecomposable quencher, and/or any other additive to the main compound and its function in the formulation.)

We would like to thank the following team for their efforts in creating these guidelines, which we hope will make improve the efficiency of our editorial review process.

Senior Editor: Steven G. Hansen

Associate Editors: Ralph R. Dammel, Roel Gronheid, and Danilo De Simone

Additional contribution: Robert Brainard

It is intended to generate, in the future, similar sets of guidelines for papers involving other topics, such as equipment, metrology, and computational methods.

(C) 2020 Society of Photo-Optical Instrumentation Engineers (SPIE) 